\title{
The University of California, San Francisco Documentation System for Retinoblastoma: Preparing to Improve Staging Methods for This Disease
}

\author{
Bertil Damato $^{\mathrm{a}} \quad$ Armin R. Afshar $^{\mathrm{a}} \quad$ Lesley Everett $^{\mathrm{a}} \quad$ Anuradha Banerjee $^{\mathrm{b}}$ \\ Steven W. Hetts ${ }^{c}$ \\ a Department of Ophthalmology, University of California, San Francisco, San Francisco, CA, USA; bepartment of \\ Pediatrics, University of California, San Francisco, San Francisco, CA, USA; ' $D$ Department of Radiology and Biomedical \\ Imaging, University of California, San Francisco, San Francisco, CA, USA
}

\section{Keywords}

Retinoblastoma $\cdot$ Treatment $\cdot$ Prognosis

\begin{abstract}
Background/Aims: Current retinoblastoma staging systems do not adequately describe the disease, especially in eyes with multiple tumors. The aims of this study were to develop methods for documenting individual tumors and to score disease burden over time. Methods: A coding system was devised to describe each tumor according to affected eye, meridian, anteroposterior location, activity, growth pattern, type of seed, and treatment. A scoring system for quantifying disease burden was developed, taking account of tumor number, size, spread, and secondary effects on the eye. $\boldsymbol{R e}$ sults: Our coding system allowed contemporaneous tumor documentation, producing datasets that enabled generation of fundus diagrams, Kaplan-Meier curves, and tables summarizing disease progression in individual tumors and eyes. Our data showed disparities between ocular and tumor documentation, e.g., indicating earlier tumor development in the left eye but younger age at presentation if disease was
\end{abstract}

\section{KARGER}

() 2018 S. Karger AG, Basel

E-Mail karger@karger.com

www.karger.com/oop worse in the right eye. Actuarial rates of local treatment failure were lower when individual tumors were analyzed than when data were reported in terms of whole eyes. Conclusion: Our methods for documenting individual retinoblastomas have facilitated the review of patients' progress in our routine practice and may provide data that could be used to refine retinoblastoma classifications in the future.

(c) 2018 S. Karger AG, Basel

\section{Introduction}

As with other diseases, full documentation of retinoblastoma is essential for planning treatment, evaluating quality of care, performing clinical trials, and predicting outcomes.

Several systems for staging retinoblastoma exist [1-3]. These have a number of limitations. First, they do not fully describe eyes harboring multiple tumors, which are often treated differently from each other and which vary in their growth pattern and response to therapy. Second, current classifications are designed to categorize disease 
at presentation, not change over time. Third, present staging systems do not reflect recent advances in intraarterial and intravitreal chemotherapy. For example, the type of vitreous seed (i.e., dust, spheres, or clouds) may be a better prognostic indicator than the distance of furthest seed from the tumor surface. Fourth, they do not take into account the number of tumors in an eye. A "group A" eye with only one tumor may not have the same prognosis as an eye with "group A" disease and multiple tumors. Finally, they do not take into account the tendency for a patient to develop new tumors after initial treatment, this tendency varying greatly between individuals. Retinoblastoma documentation needs to be more comprehensive to allow staging systems to continue to evolve in response to novel therapies and research findings.

We devised a system for coding individual tumors, also documenting tumor activity. Such a system allows the patient's history to be reviewed quickly, indicating which tumors were active most recently, how each of these was treated, and when such treatment was administered.

The aims of this study were to develop methods for documenting retinoblastoma according to each individual tumor and scoring disease burden.

\section{Patients and Methods}

The coding system was developed prospectively with data from the first 40 newly diagnosed patients treated for retinoblastoma at the University of California, San Francisco between June 2013 and December 2017. Patients were excluded if treatment had been initiated elsewhere or before June 2013, because of insufficient data. The cohort comprised 20 patients ( 9 female, 11 male) with somatic retinoblastoma and 20 patients with a germline mutation (12 female, 8 male), the latter presenting with 103 tumors in 37 eyes. Online supplementary Table 1 (see www.karger.com/doi/10.1159/000488147) summarizes baseline status, treatment, and outcomes.

The first examination under anesthesia (EUA) included: tonometry; measurement of corneal diameter; portable slit-lamp examination of the anterior chamber and lens; binocular indirect ophthalmoscopy; measurement of basal tumor diameter and thickness by B-scan ultrasonography (Eye Cubed; Ellex, Adelaide, Australia); optical coherence tomography in selected cases (Bioptigen, Durham, NC, USA); and photography with RetCam (RetCam, Pleasanton, CA, USA). Fundus drawings were prepared on an iPad tablet using drawing software (Adobe, San Jose, CA, USA) and a template designed by us and made available at www. oculonco.com (Fig. 1a, b). These drawings were uploaded into our electronic medical records as with other images and documents. Magnetic resonance imaging of the brain and orbits was performed on the same day as the first EUA or on the previous day. Subsequent EUAs included binocular indirect ophthalmoscopy, fundus photography, fundus drawings and, in selected cases, optical coherence tomography and ultrasonography.

University of California, San Francisco

Documentation of Retinoblastomas
All patients underwent investigation for germline $R B 1$ alterations. If enucleation was performed, the harvested tumor was also analyzed for RB1 alterations (Invitae, San Francisco, CA, USA; Impact Genetics, Bowmanville, ON, Canada).

Small tumors (diameter $\leq 3 \mathrm{~mm}$ ) were treated with cryotherapy if anterior and with laser if posterior. In the early part of the study, tumors considered too large for such focal therapy were treated with systemic chemotherapy if bilateral or with intra-arterial chemotherapy if unilateral; however, this changed to intra-arterial chemotherapy for all patients. Vitreous seeds unresponsive to systemic or intra-arterial chemotherapy were treated with intravitreal chemotherapy. Enucleation was reserved for group E eyes and those unresponsive to eye-conserving therapy.

Each tumor was coded with a single letter for each item according to: affected eye (left, right); meridian in clock minutes; anteroposterior tumor location (both disc and fovea [B], fovea $[\mathrm{F}]$, disc $[\mathrm{D}]$, macula not involving fovea $[\mathrm{M}]$, posterior retina not involving disc $[\mathrm{P}]$, equator $[\mathrm{E}]$, anterior retina [i.e., pre-equatorial retina] $[\mathrm{A}]$, ora $[\mathrm{O}]$, uncertain $[\mathrm{U}]$ ); and status (new [N], larger $[\mathrm{L}]$, persistent $[\mathrm{P}]$, diminished $[\mathrm{D}]$, inactive $[\mathrm{I}]$, uncertain $[\mathrm{U}]$ ). Uncertain tumor meridian was coded as 00 (e.g., when the tumor filled the eye). The disc was recorded as being involved if visible tumor extended to the disc margin or if the optic disc was obscured by the tumor. Tumors were categorized as exophytic (X), endophytic $(\mathrm{N})$, mixed $(\mathrm{M})$, vitreous seeds $(\mathrm{V})$, subretinal $(\mathrm{S})$, and uncertain $(\mathrm{U})$. We categorized tumor configuration at first visit as "mixed" if the internal limiting membrane was breached. To enable automated fundus diagrams, diffuse subretinal seeds were documented circumferentially in 5-min intervals (e.g., 5, 10, 15 , etc.), whereas with isolated subretinal seeds the actual meridian was documented. Both treated and inactive tumors were subcategorized as calcific ("cottage cheese") (1), fish flesh (2), mixed (3), flat (4), and invisible (0), in keeping with conventional practice. Regressing tumors were categorized as fish flesh (type 2) if calcifications within the tumor were minimal and nonconfluent. Vitreous seeds were subcategorized as dust (D), spheres (S), and clouds (C) [4]. Their activity was categorized as new (N), larger $(\mathrm{L})$, persistent $(\mathrm{P})$, diminished $(\mathrm{D})$, inactive $(\mathrm{I})$, and uncertain (U). Location of diffuse vitreous seeds was documented by affected quadrant (i.e., 7, 22, 37, and 52 clock minutes) whereas with isolated vitreous seeds the actual meridian was recorded, with documentation of the retinal zone when the seeds were close to the retina. Vitreous seeds were categorized as calcified (1), amorphous (2), mixed (3), and invisible (0). Treatment was coded as arterial chemotherapy (A), cryotherapy (C), enucleation (E), laser therapy (L), systemic chemotherapy (S), and vitreal chemotherapy $(\mathrm{V})$. When multiple therapeutic modalities were administered simultaneously, these were listed alphabetically. For example, a new, inferotemporal, pre-equatorial, exophytic tumor in the right eye treated with cryotherapy in a patient who also received systemic chemotherapy was coded as R35ANX,CS. Tumors were categorized as "recurrent" only if previous examination had suggested total inactivity; otherwise, they were classified as "persistent," "diminished," or "larger." Ocular morbidity, such as glaucoma, retinal detachment, and uveitis were also recorded.

Eyes were scored as shown in Table 1 so as to indicate the disease burden at each examination.

Findings were documented in our hospital's electronic medical records (APeX; EPIC Systems, Verona, WI, USA). After 2013 these were entered into document flowsheets, which we customized to 

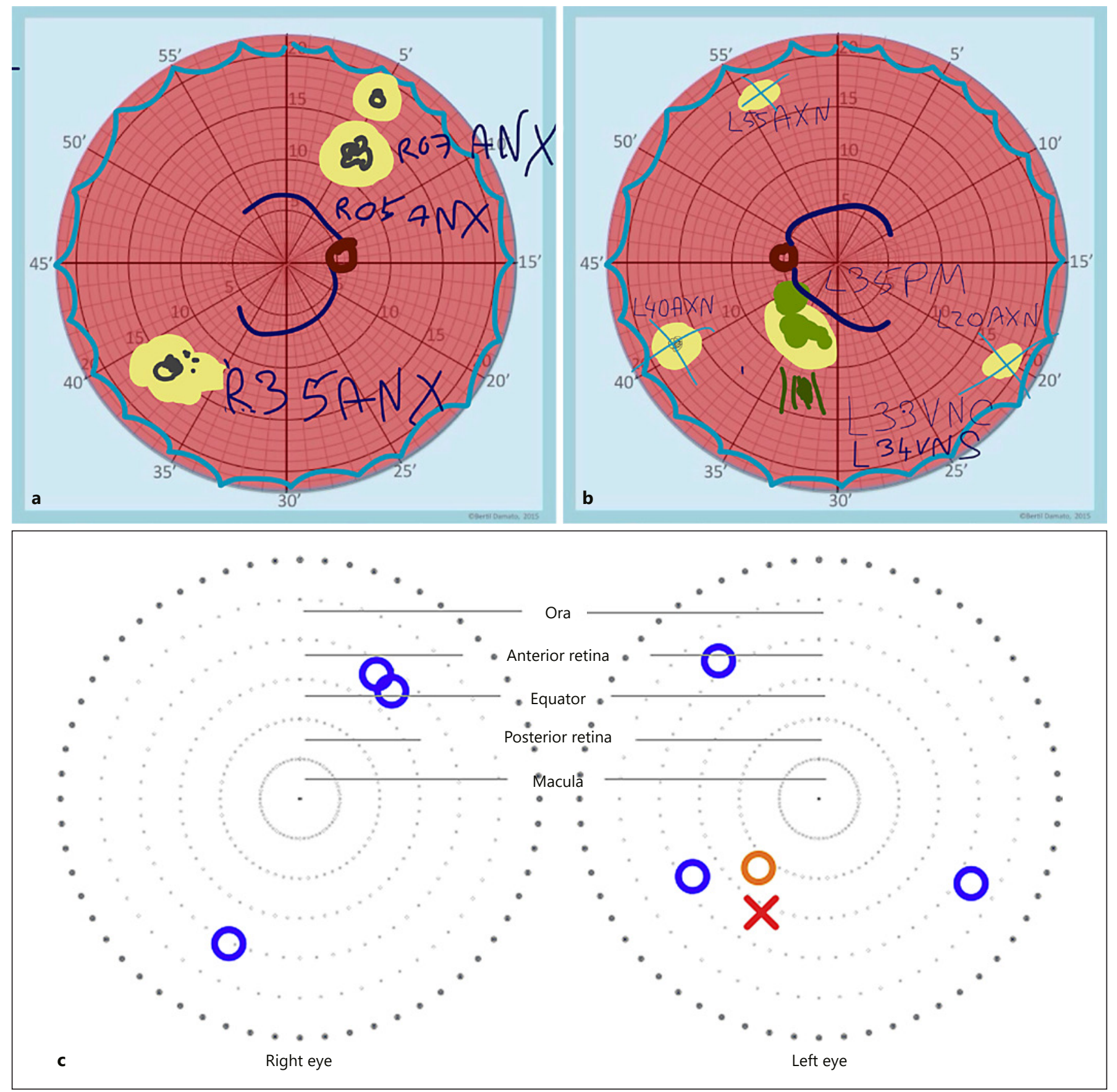

Fig. 1. Multiple retinoblastomas in both eyes of an African-American baby boy. a, b Fundus drawings of the right (a) and left eye (b), prepared on a tablet during the examination under anesthesia, depicting exophytic tumors as light green, endophytic tumors as

serve as a registry. Data were exported to SPSS (IBM SPSS Statistics for Macintosh, Version 22.0, Armonk, NY, USA) for statistical analysis. Fundus diagrams were generated automatically from numerical data using Stata (Release 14.2 for Mac; StataCorp, College medium green, and vitreous seeds as dark green. c Fundus diagram, generated with Stata, depicting exophytic tumors in blue, endophytic tumors in orange, and vitreous seeds in red. The text was added using PowerPoint. 
Table 1. Tentative system for scoring eyes and individual tumors to categorize retinoblastoma-affected eyes into groupings similar to those of the IRC

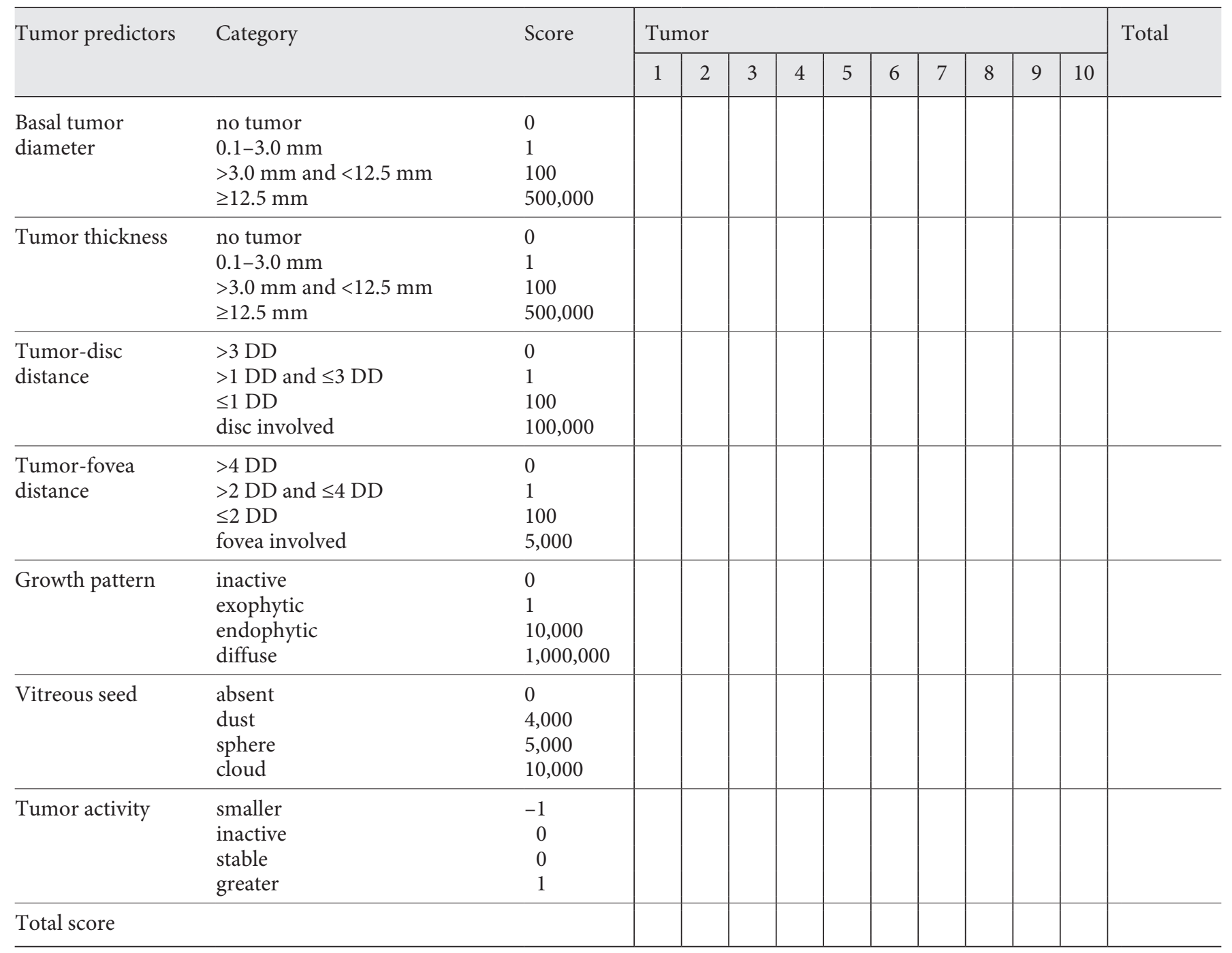

(Table continued on next page.)

\section{Results}

Figure 2 shows how our coding system enables documentation of tumor locations within each eye. For example, Figure 2a demonstrates how tumors were more commonly medial and how those detected at a later age tended to be more anterior. Figure $2 \mathrm{~b}$ shows the location of new tumors developing after completion of intra-arterial chemotherapy in patients with germline disease.

Figure 3 shows how this coding system would facilitate investigation of age at detection of each tumor. For example, in a cohort of 55 germline retinoblastomas detected after the first examination, such analysis indicated that postequatorial tumors are detected at a younger age than anterior tumors (Fig. 3a). Figure 3 b suggests that tumors in the left eye tend to be detected at a younger age than those in the right eye. This result is different from that of Figure $3 \mathrm{c}$, which suggests that patients presented at a younger age when the disease was more advanced in the right eye.

As expected, the reported recurrence rates were lower when described in terms of tumors than in terms of eyes. Kaplan-Meier analysis indicated that recurrences occurred in $29.9 \%$ of 61 eyes that had not undergone primary enucleation. These 61 eyes harbored 156 tumors, $11.1 \%$ of which recurred. 
Table 1 (continued)

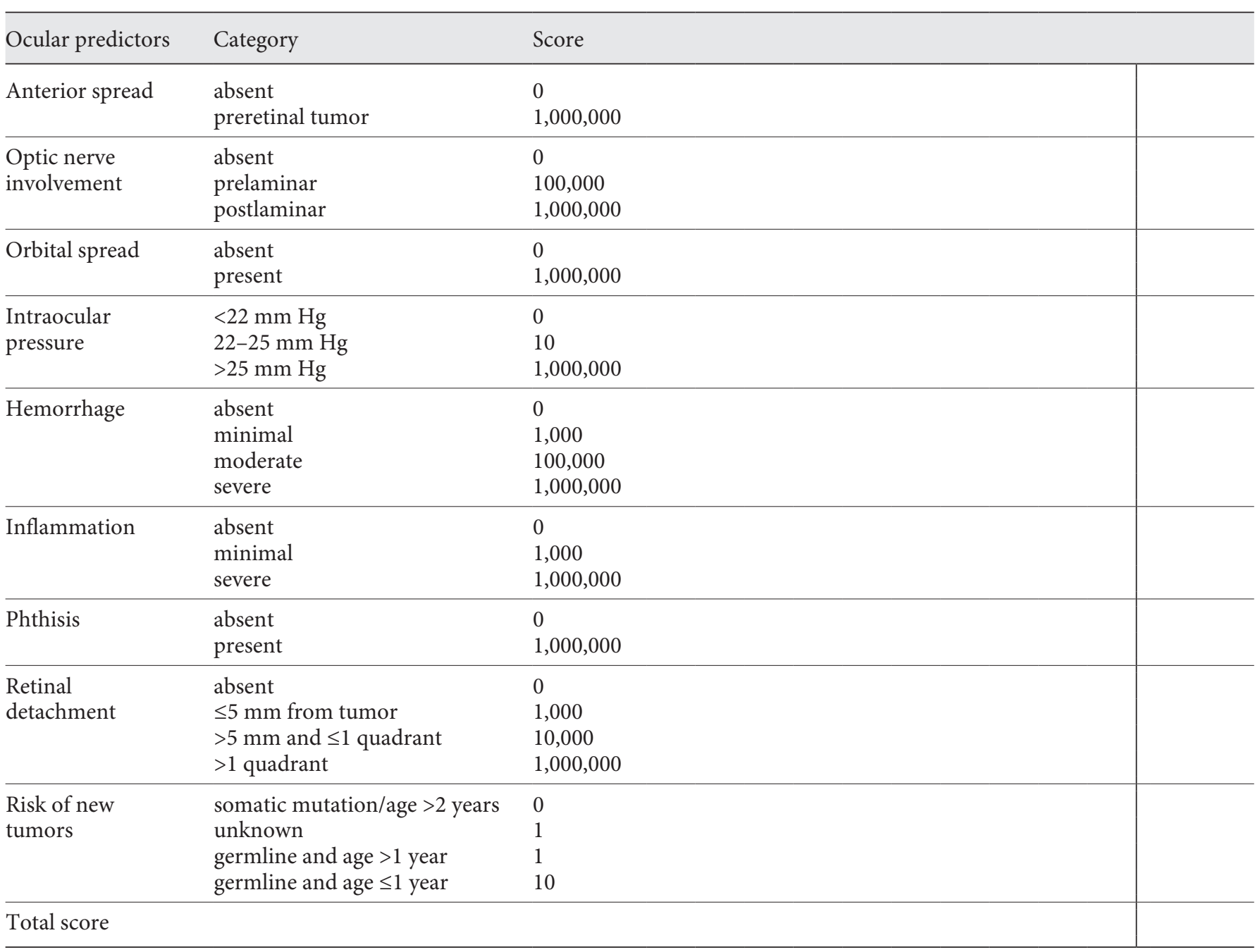

Approximate IRC groups: $1-99=\mathrm{A} ; 100-999=\mathrm{B} ; 1,000-9,999=\mathrm{C} ; 10,000-99,999=\mathrm{D} ; \geq 1,000,000=\mathrm{E}$. IRC, International Retinoblastoma Classification.

Table 2 shows how our coding system aids detailed review of patients' previous treatment, providing information on the location, growth pattern, treatment, and outcome of each tumor at every examination. It summarizes the history of a baby girl presenting with bilateral retinoblastoma at the age of 6 months. The right eye was enucleated after one dose of systemic neoadjuvant chemotherapy. The left eye was found to have five exophytic retinal tumors and two tumors that had perforated the internal limiting membrane, with dusty vitreous seeding inferotemporally and medially. The patient received three doses of systemic chemotherapy in addition to laser therapy to most lesions. At the age of 12 months, a new exophyt- ic tumor was noted in the inferotemporal equatorial retina and was treated with laser. At the age of 15 months, a suspicious lesion in the inferonasal, postequatorial retina was lasered as a precaution. At the age of 14 months, two recurrences, consisting of fish flesh and calcification, were noted in the temporal and inferotemporal equatorial region of the left eye and were treated with laser, the inferotemporallesion in the 20-min meridian ("4 o'clock") subsequently receiving cryotherapy and laser treatment. Another recurrence, located in the horizontal, medial, equatorial region, was diagnosed at the age of 24 months and was treated with laser therapy and cryotherapy. No further tumor activity was noted over the next 10 months, 
Fig. 2. Computer-generated fundus diagrams showing germline tumor locations according to age when new tumors were detected, with red circles, orange diamonds, green triangles, empty purple squares, and filled purple squares representing tumors found at 0-6 months, $>6-12$ months, $>12-18$ months, $>18-24$ months, and $>24$ months of age, respectively (a), and the location of new germline tumors developing after completion of intra-arterial chemotherapy (b). The numbers in red indicate locations and case numbers of new tumors after completion of intra-arterial chemotherapy. The blue circles indicate new tumors developing before completion of intra-arterial chemotherapy and those developing in eyes without this treatment.

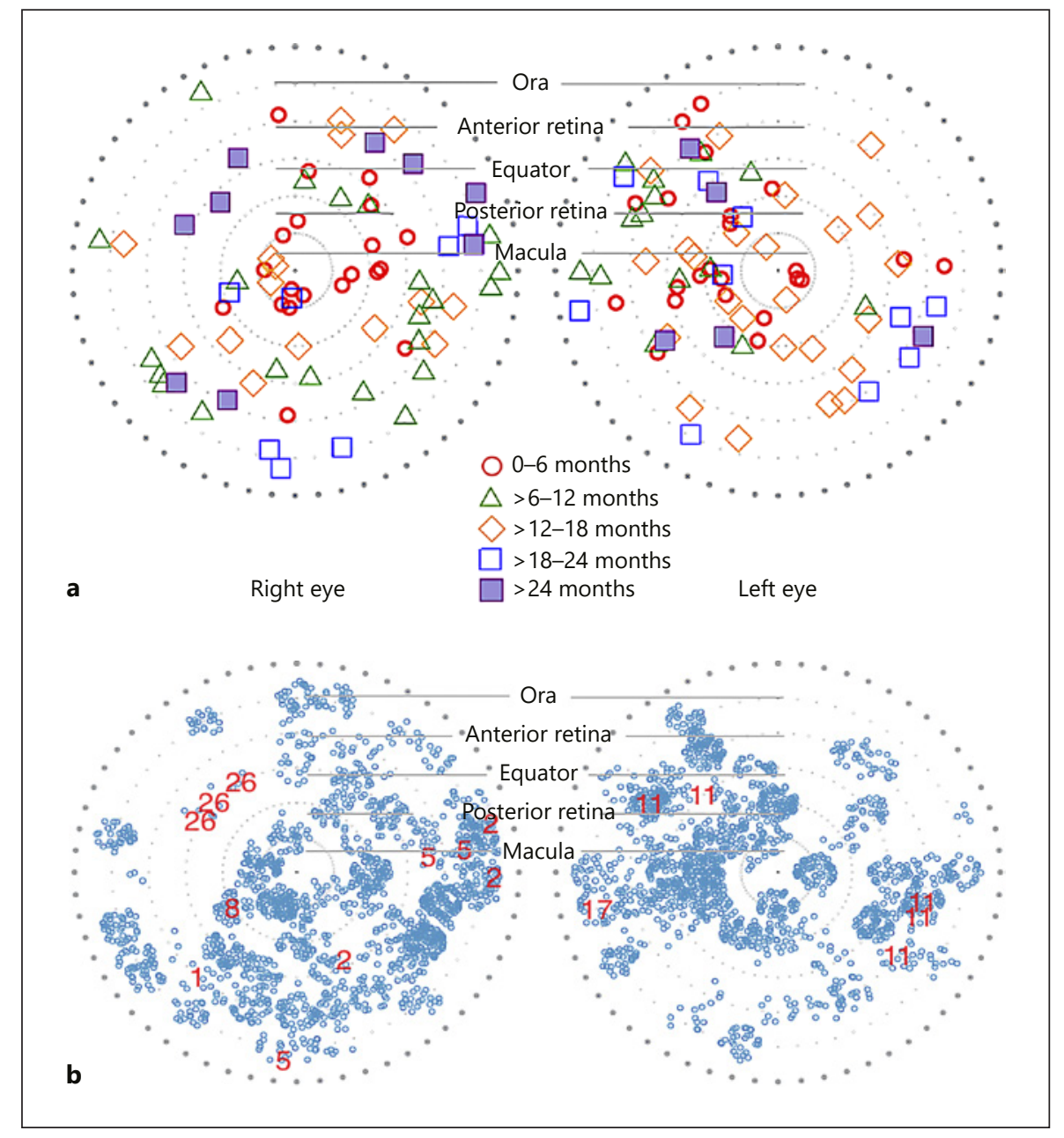

until shortly before the closing of the study. This table also shows how scores varied with disease burden.

\section{Discussion}

\section{Main Outcomes}

We have devised a system for coding the location and behavior of individual tumors in retinoblastoma-affected eyes. We have also developed a method for scoring disease burden in affected eyes and for documenting change over time.

\section{Strengths and Weaknesses}

Our treatment selection shifted from systemic to intraarterial chemotherapy during this project; however, since we were not evaluating therapy, this did not detract from our study.

University of California, San Francisco

Documentation of Retinoblastomas

\section{Methods}

Drawings were made on a tablet because it was easier to see the images in the darkened room and helped to avoid fragments from color pencils and erasers from contaminating the operative field. Further, these digital images aided oral communication with the patients' relatives immediately after each EUA and were easily uploaded into electronic health records and incorporated into reports, which were copied to the parents.

In our scoring system, predictive factors were categorized and scored to derive groupings similar to current International Retinoblastoma Classification (IRC) systems. However, continuous data were nevertheless documented to enable future development of tumor staging. For example, IRC systems do not differentiate between tumors that are $3 \mathrm{~mm}$ thick and those that have a thickness of, say, $10 \mathrm{~mm}$. Tumor diameter and thickness were documented separately because following treatment, tu- 


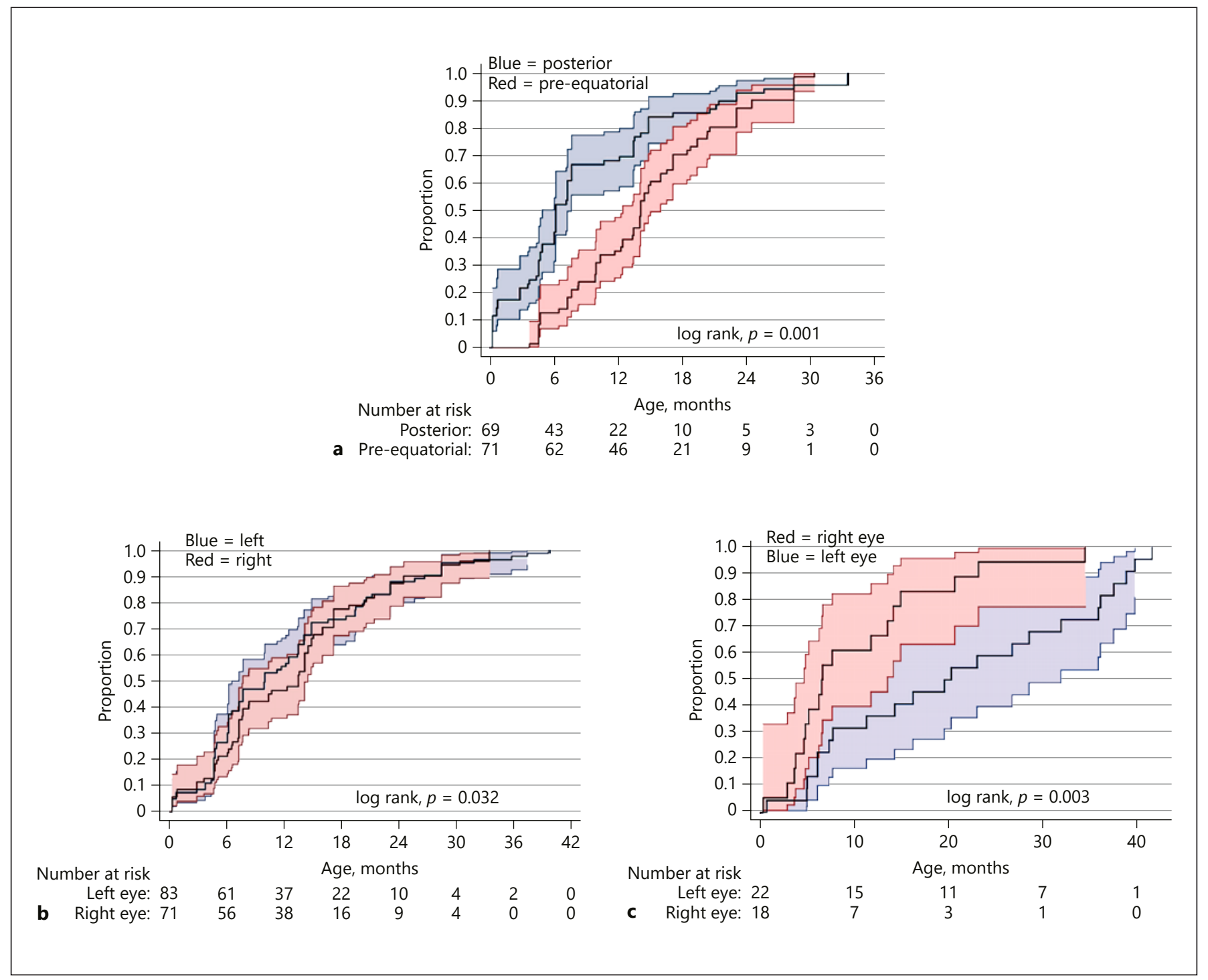

Fig. 3. a-c Age at detection of each new germline tumor after the first examination, according to anteroposterior tumor location (a), tumor laterality (b), and worst affected eye (c).

mor thickness may regress differently from tumor base. We did not use tumor volume in our system because its estimation was subjective and because it was related to ocular volume, which was not measured; therefore, we preferred to rely on tumor thickness. Although tumor dimensions and distances from disc and fovea were documented, these were not included in the tumor codes to avoid such codes from becoming unwieldy. Tumor growth and regression were mostly categorized according to ophthalmoscopic appearances; this is in keeping with conventional practice, because ultrasonographic measurements of every tumor are not routinely obtained in follow-up EUAs. Circumferential tumor localization was documented in clock minutes because these were easier to image mentally than degrees and more precise than clock hours.

Vitreous seeds were categorized by type because these vary in their chemoresponsiveness. Locations of vitreous and subretinal seeds were categorized by quadrant and clock hour, respectively, to enable computer-generated fundus diagrams. Further studies are required to refine seed categorization, ideally describing severity more fully.

Our system also allowed documentation of other factors, such as glaucoma, uveitis, retinal detachment, extra- 


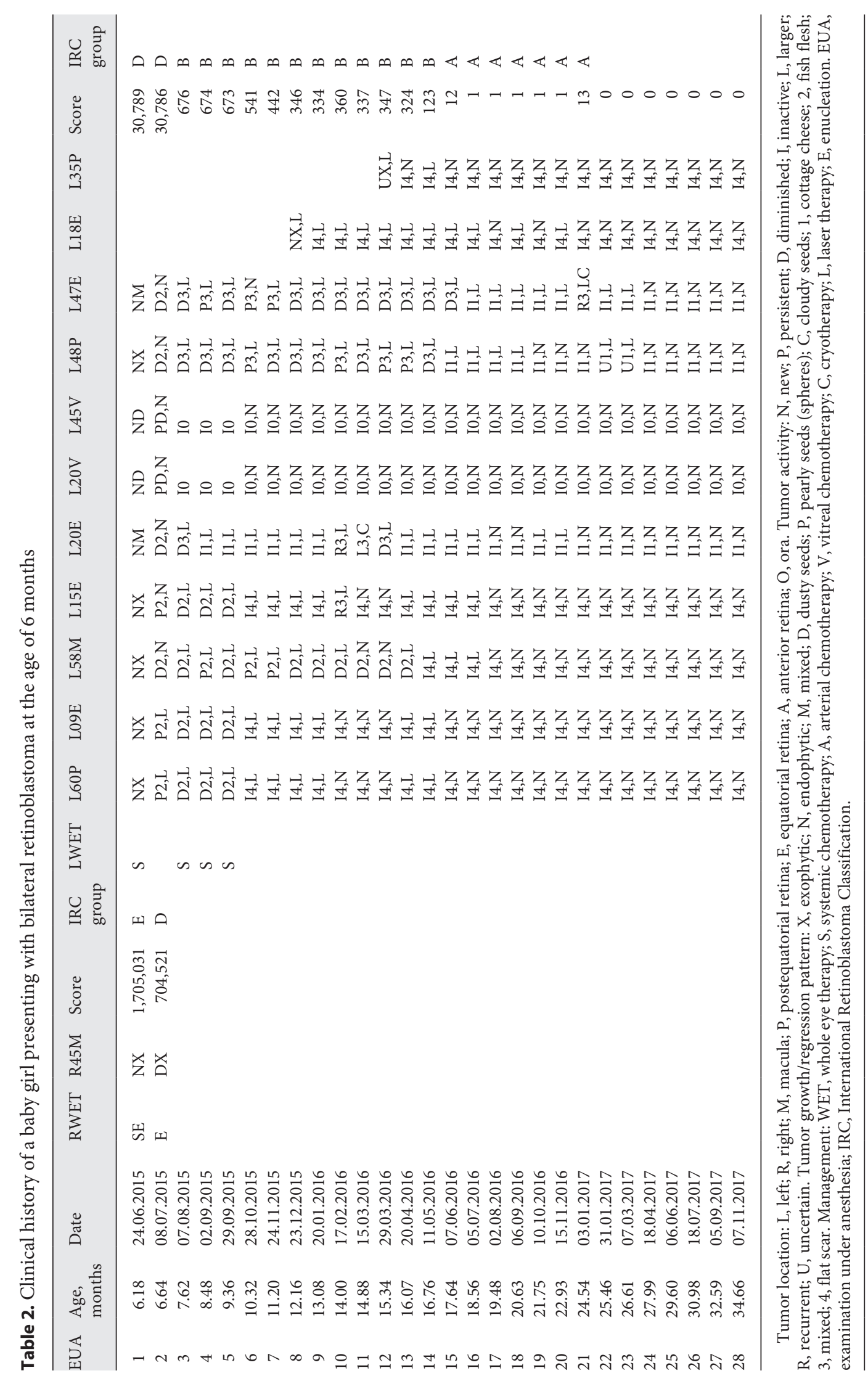


ocular spread, and optic nerve involvement. We categorized intraocular pressure only according to tonometry, without including "buphthalmos" because this was secondary to intraocular pressure. Retinal detachment was recorded as a continuous variable to enable future development.

\section{Results}

The clinical results reported in this article are intended to demonstrate the potential of our coding system for retinoblastoma and not to make any inferences regarding therapeutic efficacy.

Table 2 shows how the entire ocular history can be summarized in one table so that it is possible for the clinician to quickly review each tumor individually. If the patient had glaucoma, extraocular spread, or other adverse factors, these would have been listed in an additional column. Table 2 also shows how the disease stage improved over time; however, it also shows how the scores varied widely within the IRC B grouping, which therefore does not seem to describe the disease burden precisely. In examinations $16-20$, the disease in the left eye was categorized as "A" in the absence of any viable tumors, and this was meant to reflect some risk of new tumor formation. This was done to emulate the TNM staging system but requires further evaluation [2].

Kaplan-Meier analyses suggest that in eyes with multiple tumors, local tumor control is described more precisely when outcomes are reported by tumor than by eye.

Our system for localizing tumors demonstrated a higher prevalence of medial tumors, which has been reported previously [5]. This is in keeping with the higher density of cones in the medial retina and evidence that retinoblastomas arise from cone precursors $[6,7]$.

\section{Further Studies}

Our coding system is intended only as a method for documenting retinoblastoma in greater detail so as to provide data that might be useful in the future to any groups or individuals striving to improve existing classifications so that these do not lose relevance when new therapies are developed (e.g., intravitreal chemotherapy). It is not the aim of this study to develop or propose a new classification.

As mentioned, our scoring system is designed to categorize affected eyes into groupings similar to those of the IRC. We are currently evaluating it in a larger cohort of patients. There is scope for further studies to adjust the scoring of each risk factor according to the outcome this factor is intended to predict (i.e., visual acuity, ocular conservation, mortality). For example, foveal involvement should predict visual acuity but not patient survival. There is also a need to determine whether our approach usefully allows prognostication to be revised according to the findings of each successive examination, as well as the patient's age and type of mutation. It is hoped that our proposals will be further refined and validated with data from other centers, following standardized protocols [8]. Prospects for such studies would be improved by the development of software programs enabling clinicians to collect data contemporaneously, effortlessly, efficiently, and accurately.

\section{Conclusions}

We have developed a coding system for documenting individual tumors and scoring disease burden in eyes affected by retinoblastoma. Further studies are needed to enhance the ergonomics of this system and to validate it in other centers.

\section{Acknowledgments}

The authors acknowledge the contributions of Greg Bever, Andrew Kao, Joyce Meza, and Michael Seider, who assisted with data collection and documentation, Kelly Babic for imaging, and Vaishali Doshi, who computerized much of the data. We also gratefully acknowledge financial support from the Giannini Fund under the auspices of That Man May See. This work was made possible in part by NIH-NEI EY002162 - Core Grant for Vision Research and by a Research to Prevent Blindness unrestricted grant.

\section{Statement of Ethics}

This study complied with the Health Insurance Portability and Accountability Act (HIPAA). Approval of the UCSF Institutional Review Board was obtained (No. 16-19878).

\section{Disclosure Statement}

The authors have no conflicts of interest to declare. 


\section{References}

1 Murphree AL, Chantada GL: Retinoblastoma: staging and grouping; in Singh AD, Murphree AL, Damato BE (eds): Clinical Ophthalmic Oncology: Retinoblastoma, ed 2. Heidelberg, Springer, 2015, pp 29-38.

2 Mallipatna AC, Gallie BL, Chevez-Barrios P, Lumbroso-Le Rouic L, Chantada GL, Doz F, Brisse HJ, Munier FL, Albert DM, CatalaMora J, Desjardins L, Suzuki S, Carroll WL, Coupland SE, Finger PT: Retinoblastoma; in Amin MB (ed): AJCC Cancer Staging Manual, ed 8. New York, Springer, 2016, pp 819831 .
3 Shields CL, Shields JA: Basic understanding of current classification and management of retinoblastoma. Curr Opin Ophthalmol 2006; 17:228-234.

$\checkmark 4$ Francis JH, Abramson DH, Gaillard MC, Marr BP, Beck-Popovic M, Munier FL: The classification of vitreous seeds in retinoblastoma and response to intravitreal melphalan. Ophthalmology 2015;122:1173-1179.

5 Munier FL, Balmer A, van Melle G, Gailloud C: Radial asymmetry in the topography of retinoblastoma. Clues to the cell of origin. Ophthalmic Genet 1994;15:101-106.

-6 Curcio CA, Sloan KR, Kalina RE, Hendrickson AE: Human photoreceptor topography. J Comp Neurol 1990;292:497-523.
7 Xu XL, Singh HP, Wang L, Qi DL, Poulos BK, Abramson DH, Jhanwar SC, Cobrinik D: Rb suppresses human cone-precursor-derived retinoblastoma tumours. Nature 2014;514: 385-388.

8 Kattan MW, Hess KR, Amin MB, Lu Y, Moons KG, Gershenwald JE, Gimotty PA, Guinney JH, Halabi S, Lazar AJ, Mahar AL, Patel T, Sargent DJ, Weiser MR, Compton C; members of the AJCC Precision Medicine Core: American Joint Committee on Cancer acceptance criteria for inclusion of risk models for individualized prognosis in the practice of precision medicine. CA Cancer J Clin 2016; 66:370-374. 